\title{
Noise dependence with pile-up in the ATLAS Tile calorimeter
}

\author{
J. P. Araque \\ on behalf of the ATLAS Tile Calorimeter System
}
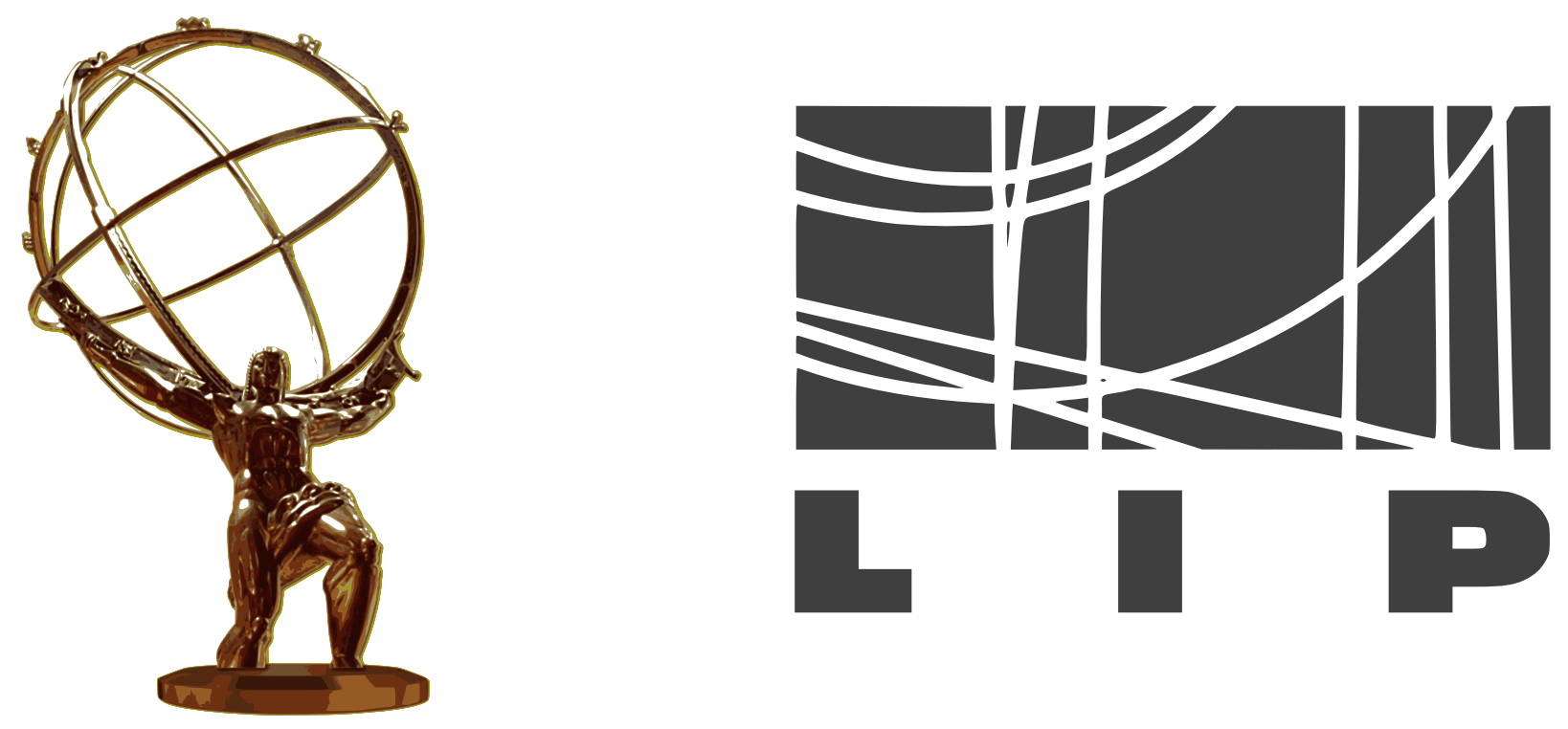

ANIMMA 2014

Lisbon, 20-24 April 2015 


\section{The Tile Calorimeter}

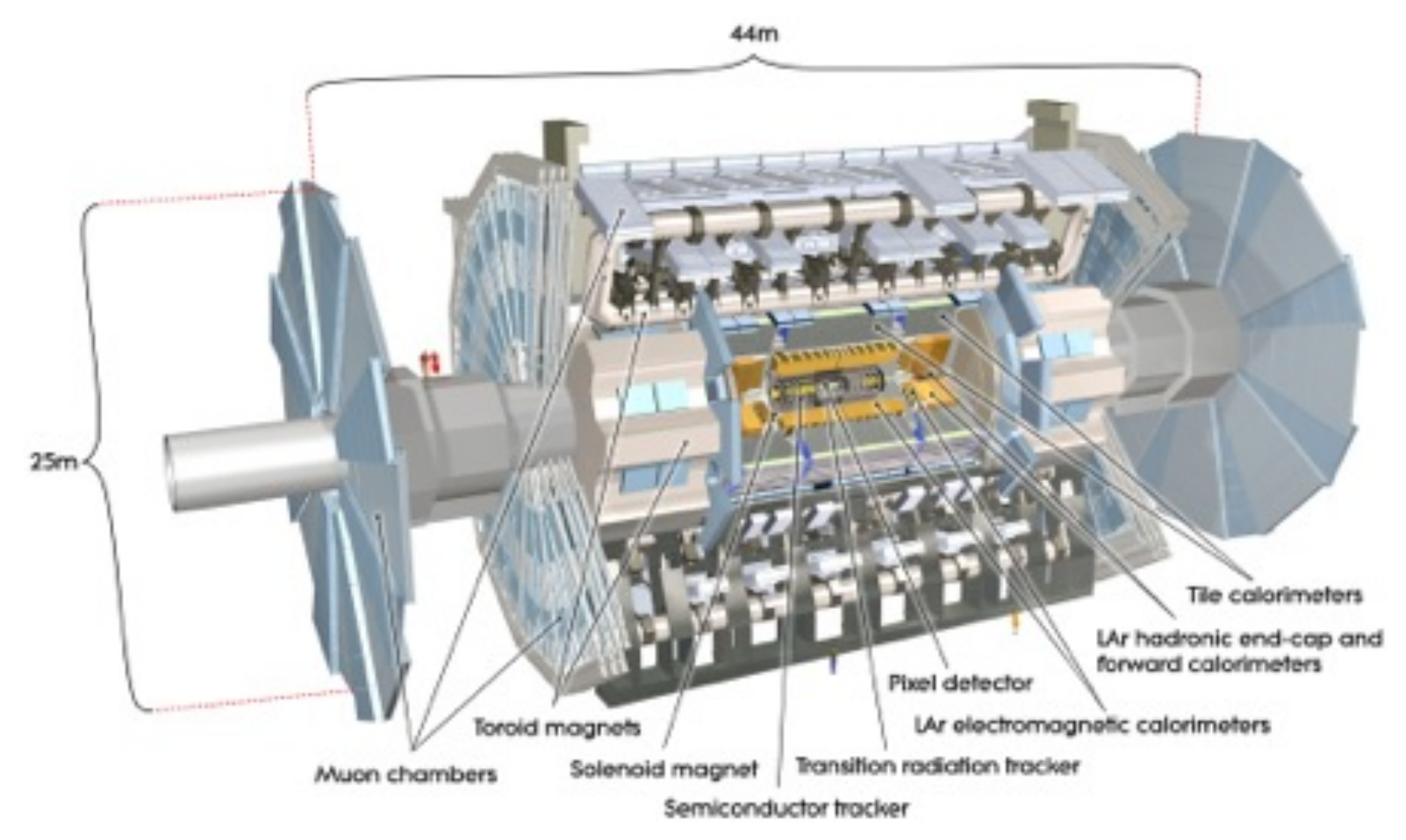

- $\quad$ The Tile Calorimeter is the central hadronic calorimeter of the ATLAS detector.

- Composed of iron layers (as passive material) and scintillating plastic layers (as active material).

- Structure:

- 4 partitions (LBA, LBC,EBA and EBC).

- 4 layers (A,BC,D and special layers).

- 16 towers (steps of 0.1 in $\eta$ ).

- 64 modules (divisions in the azimuthal angle $\phi)$.

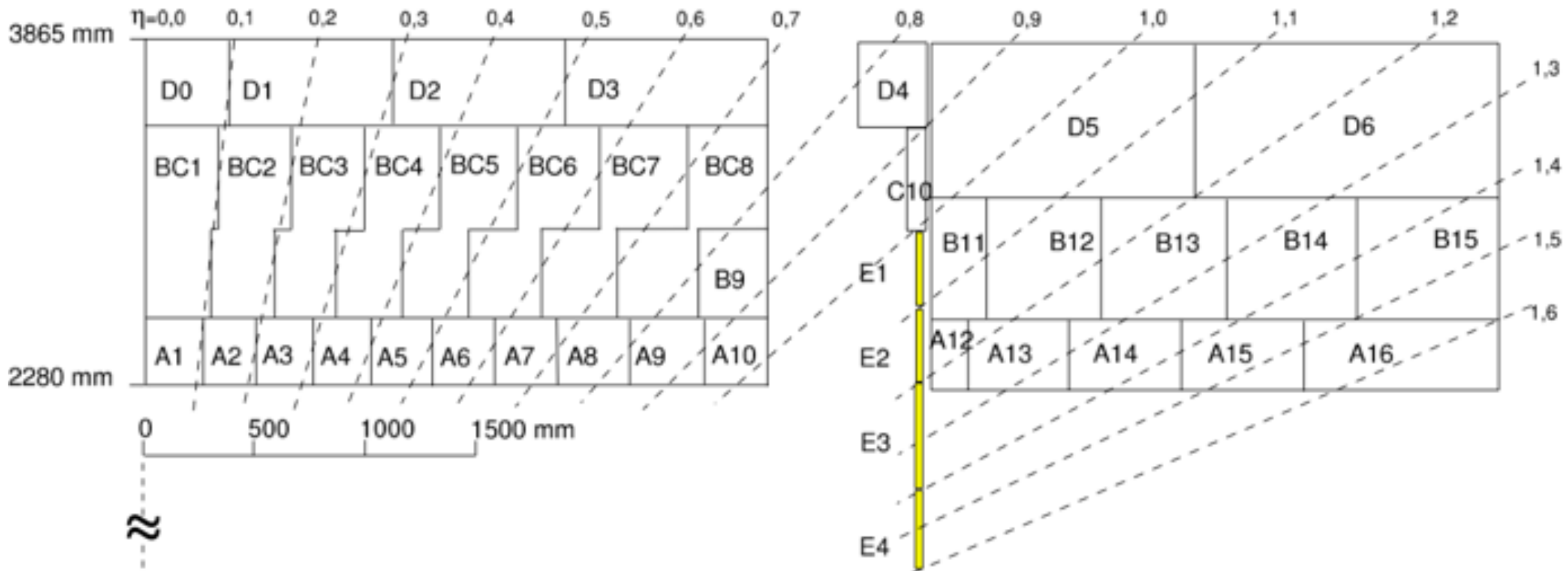




\section{Pile-up in the ATLAS detector}
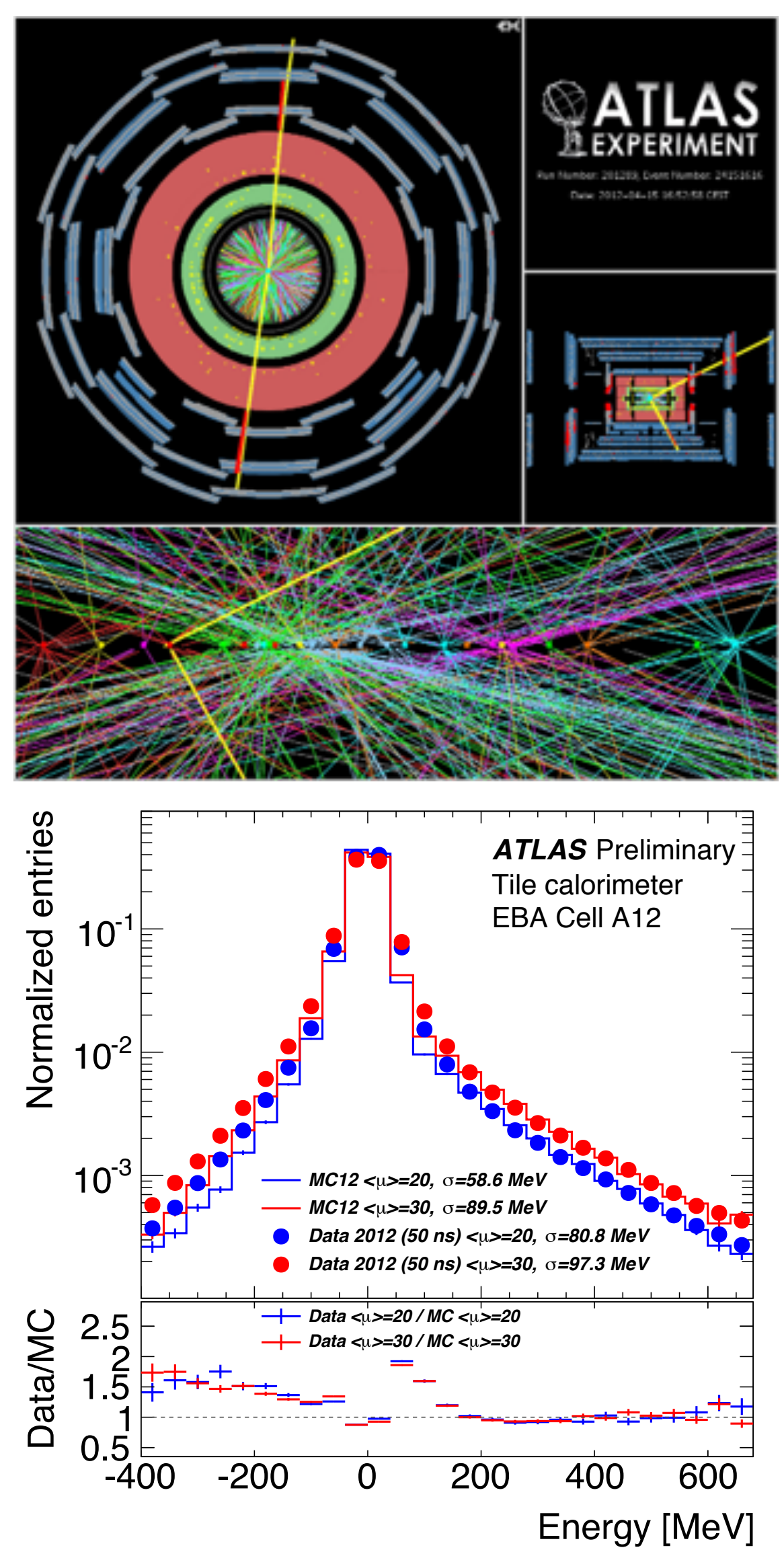

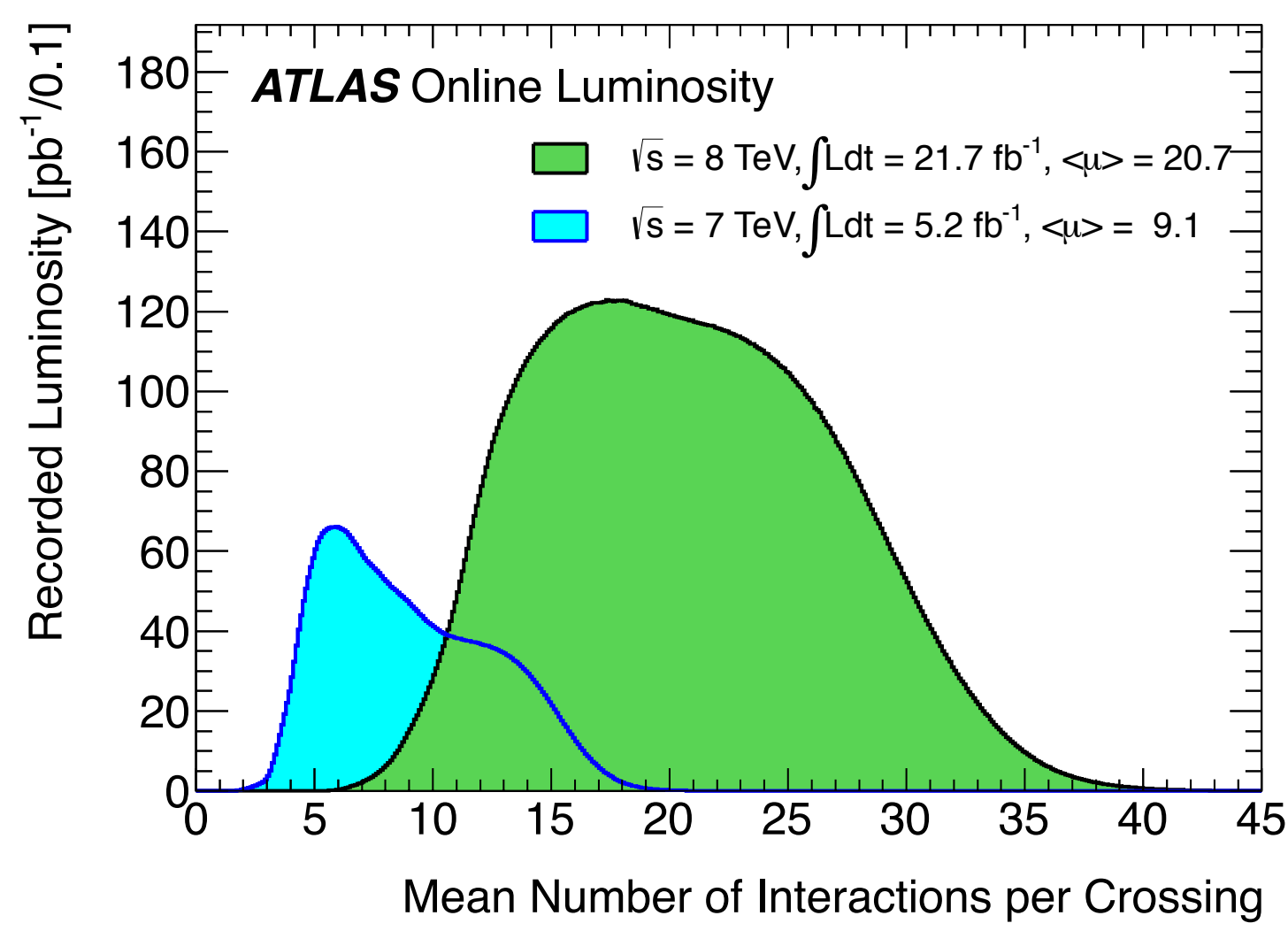

- Tens of proton-proton interactions take place in a bunch crossing inside the ATLAS detector.

- Pile-up events also deposit energy in the TileCal cells which is hard to distinguish from the energy deposited by the interesting event.

- This is known as pile-up noise and is a component to the cell noise measured that increases as pile-up increases. 


\section{RMS as the noise estimator}

- The RMS of the energy distribution can be used as a noise estimator:

$$
\sqrt{\left\langle E^{2}\right\rangle-\langle E\rangle^{2}}
$$

- The noise measured increases with the mean number of interactions per bunch-crossing.

- The bunch-spacing also increases the cell noise.
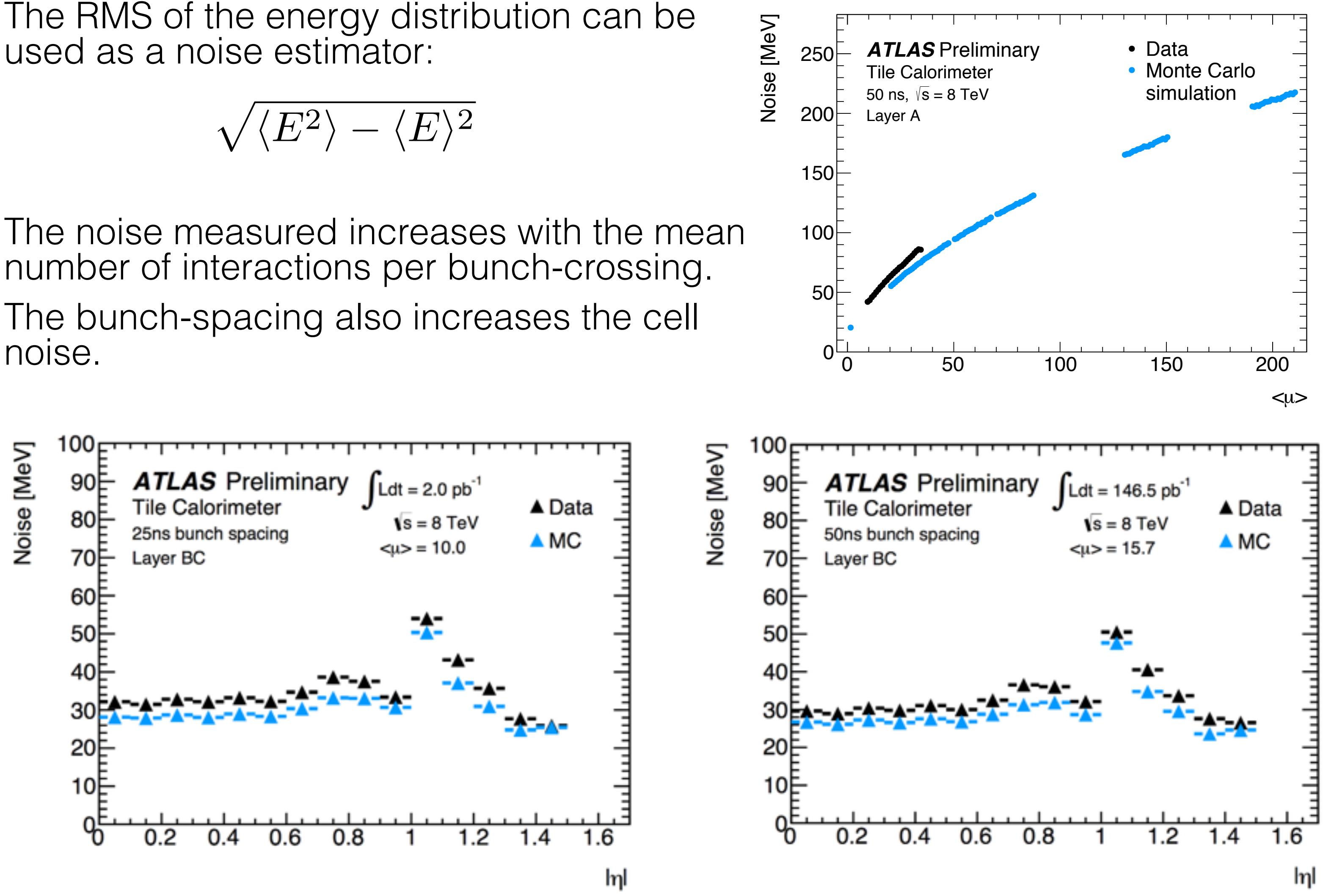


\section{Quantiles of the energy distribution as the noise estimator}

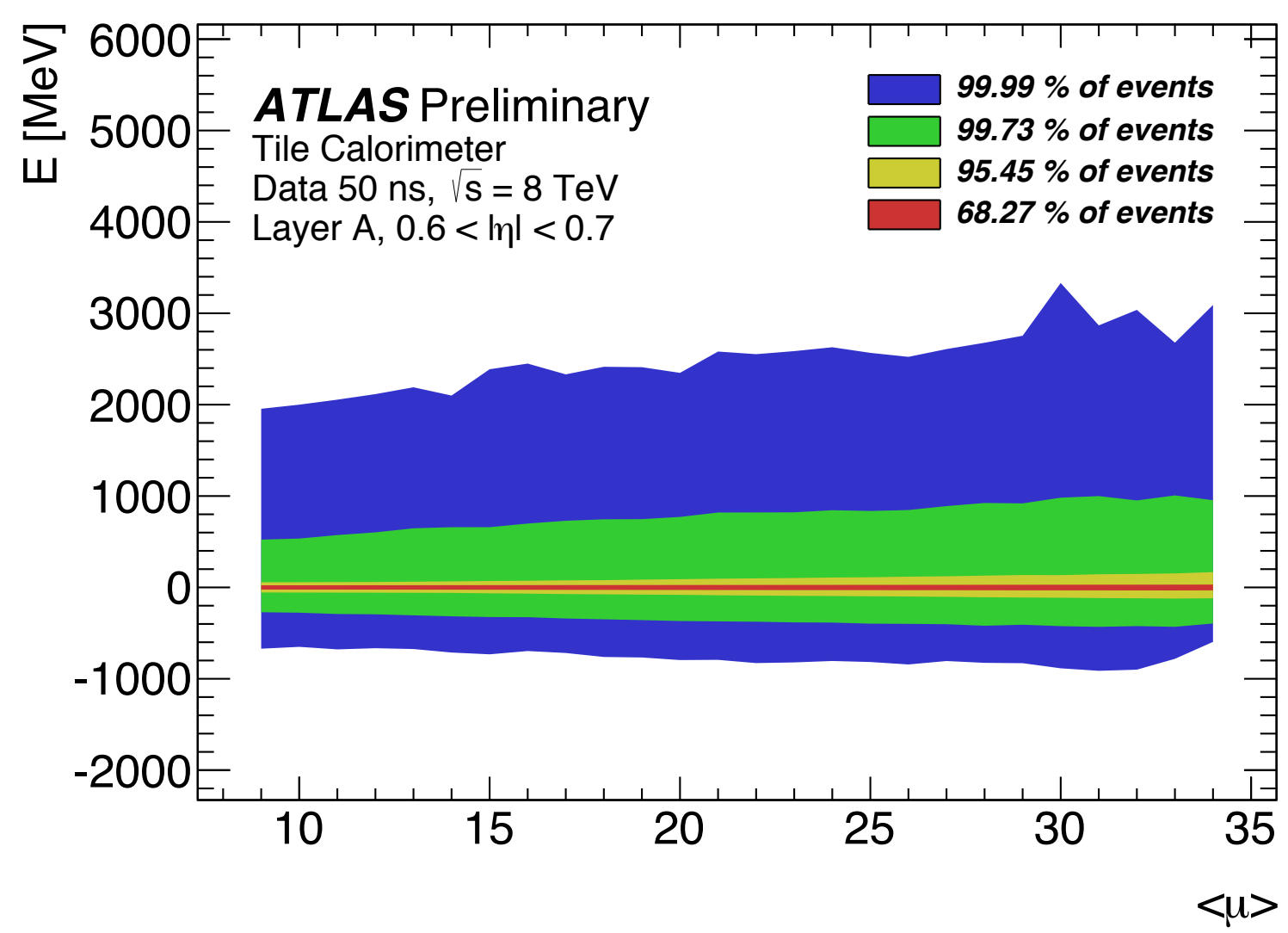

- The quantiles of the energy distribution can be used to better characterise its shape.

- The energy $\varepsilon$ is the $k_{\text {th }}$ quantile of the energy distribution with $Q$ quantiles if:

$$
P(E \leq \epsilon)=k / Q \text {. }
$$

- Non-gaussian behaviour with larger positive tails.
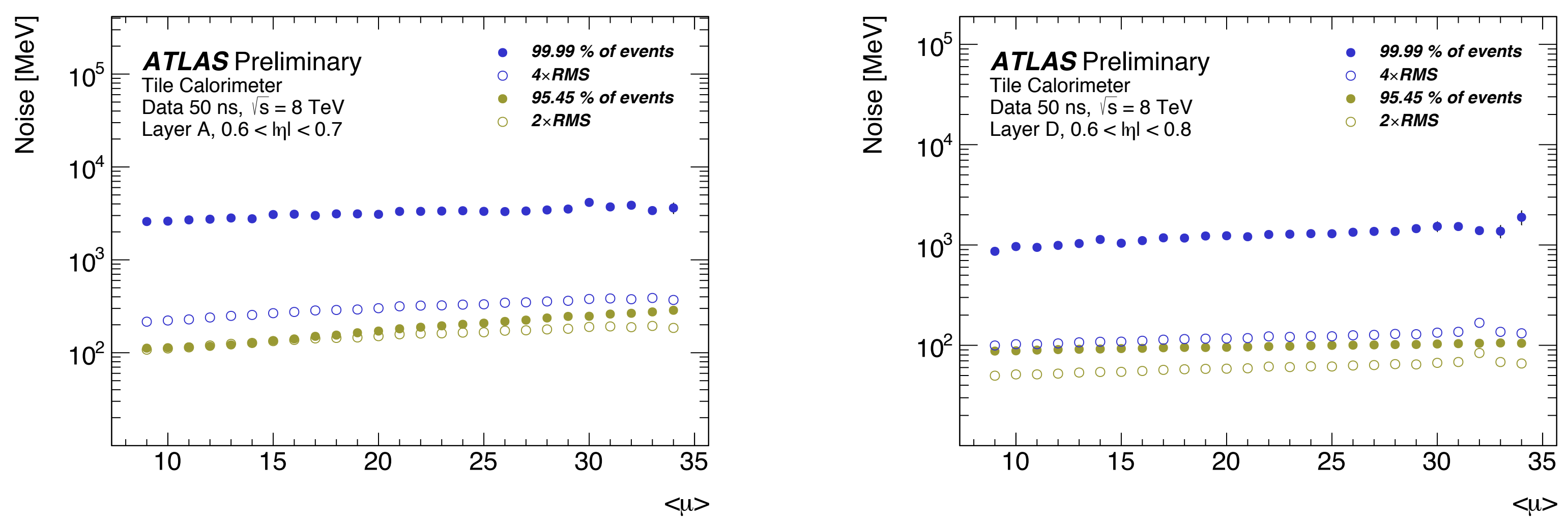


\section{Conclusions}

- The high rate at which the LHC produces proton-proton collisions implies a high amount of pile-up events taking place in the ATLAS detector.

- In the second operation phase of the LHC, with higher energy (13 TeV) and smaller bunch-spacing (25 ns), pile-up is expected to increase.

- To study the TileCal cell noise as a function of pile-up two estimators have been presented:

- The RMS of the energy distribution.

- The quantiles of the energy distribution.

- Using the quantiles estimator the shape of the energy distribution can be better characterised. 